\title{
Existence Results for a Michaud Fractional, Nonlocal, and Randomly Position Structured Fragmentation Model
}

\author{
Emile Franc Doungmo Goufo, Riëtte Maritz, and Stella Mugisha \\ Department of Mathematical Sciences, University of South Africa, Florida Science Campus, Florida 0003, South Africa \\ Correspondence should be addressed to Emile Franc Doungmo Goufo; dgoufef@unisa.ac.za
}

Received 12 May 2014; Accepted 28 May 2014; Published 12 June 2014

Academic Editor: Abdon Atangana

Copyright ( 2014 Emile Franc Doungmo Goufo et al. This is an open access article distributed under the Creative Commons Attribution License, which permits unrestricted use, distribution, and reproduction in any medium, provided the original work is properly cited.

\begin{abstract}
Until now, classical models of clusters' fission remain unable to fully explain strange phenomena like the phenomenon of shattering (Ziff and McGrady, 1987) and the sudden appearance of infinitely many particles in some systems having initial finite number of particles. That is why there is a need to extend classical models to models with fractional derivative order and use new and various techniques to analyze them. In this paper, we prove the existence of strongly continuous solution operators for nonlocal fragmentation models with Michaud time derivative of fractional order (Samko et al., 1993). We focus on the case where the splitting rate is dependent on size and position and where new particles generating from fragmentation are distributed in space randomly according to some probability density. In the analysis, we make use of the substochastic semigroup theory, the subordination principle for differential equations of fractional order (Prüss, 1993, Bazhlekova, 2000), the analogy of Hille-Yosida theorem for fractional model (Prüss, 1993), and useful properties of Mittag-Leffler relaxation function (Berberan-Santos, 2005). We are then able to show that the solution operator to the full model is positive and contractive.
\end{abstract}

\section{Model's Motivation and Introduction}

The dynamics of clusters' fragmentation occurs in many branches of natural sciences ranging from physics, through chemistry, engineering, biology, ecology, and numerous domains of applied sciences, such as the depolymerization, the rock fractures, and breakage of droplets. There exists a vast literature on classical fragmentation models and many of them have been deeply analyzed in different works (see, e.g., [1-8]) where authors investigated their conservativeness, honesty, and existence of solutions. However, investigations on fractional versions of these models are still yet to come.

The concepts of fractional derivatives and fractional integral started in 1695 when L'Hospital questioned about the meaning of the operator $d^{n} y / d x^{n}$ if $n=1 / 2$; that is, "what if $n$ is fractional?" Leibniz then replied as " $d^{1 / 2} x / d x^{1 / 2}$ will be equal to $x \sqrt{d x: x}$." Then, there is a growing interest in extending the normal calculus with integer orders to noninteger orders (real or complex order) [9-14] because its applications have attracted a great range of attention in the past few years. As an example, fractional evolution equations are used to model many problems in applied sciences, engineering, and technology including applied mathematics, computing, physics, biology, chemistry, and economics. For example, in [13], the authors used the concept of the variational order derivative together with Crank-Nicholson technique to provide a generalization of the groundwater flow equation, solve it, and present a numerical solution. In [14], two methods including Frobenius and Adomian decomposition method were used to generalize the classical Darcy law by regarding the water flow as a function of a noninteger order derivative of the piezometric head. In the process, systems with derivative of fractional order have become a useful tool for describing nonlinear phenomena occurring in epidemiological models and spatial and temporal spreads.

Many processes in real life are described by classical models taking the form of Cauchy problems given by

$$
\frac{d}{d t} g(t)=\mathscr{P} g(t)
$$


subject to initial condition

$$
g\left(t_{0}\right)=0, \quad t_{0}<t
$$

where $t \in \mathbb{R}_{+}$is the time and $\mathscr{P}$ is an operator in a Banach space. The aim here is to find the state $g(t)$ of the system at a time $t>t_{0}$ depending on the initial state. Hence, at an infinitesimal and bounded scale, the rate of accumulation or loss of matter in the system is characterized by the classical derivative $d / d t$. This leads us to three essential motivations for considering, in this paper, the fractional nonlocal and randomly position structured fragmentation model. The following motivations are the cause of increasing eagerness among scientists to extend well known old models to models with fractional derivative, in order to investigate them with various and different methods. The aim is to try new techniques hoping to establish broader outlooks on the real phenomena these differential evolutions equations describe.

First Motivation. Classical fragmentation processes are difficult to analyze as they involve the evolution of two intertwined quantities: the mass of the ensemble and the number of particles in it. That is why, though linear, they display nonlinear features such as phase transition which, in this case, is called shattering. Indeed, classical models of clusters' fission with normal derivative $d / d t$ cannot fully explain these unusual phenomena, which include not only the phenomenon of shattering, but also the sudden appearance of an infinite number of particles in some systems which contained at the beginning a finite particles number [15]. Shattering is seen as an explosive or dishonest Markov process; see, for example, $[16,17]$, and it has been associated with an infinite cascade of breakup events creating a "dust" of particles of zero size which, however, carry nonzero mass.

Second Motivation. We mentioned that $d / d t$ can be associated with the representation of the rate of change (accumulation or loss) in the system, considered at infinitesimal bounded space. But, this is not always true since the rate of accumulation or loss can be different from $d / d t$. Indeed, most of the infinitesimal spaces for complex models can contain hindering or obstacles (of various sizes) where the variable under study is temporarily parked or stuck. In this condition, the classical $d / d t$ will no longer replicate, with certitude, the real picture of accumulation or loss. In the same way, these obstacles could be holes or forbidden zones in the infinitesimal space where the variable (particle, mass, density, flux, etc.) cannot reside. Hence, the fractional differentiation $D_{t}^{\alpha}$, with $\alpha \in \mathbb{R}$ or $\mathbb{C}$, may give the sub- or superrate of accumulation or loss with index $\alpha$ representing the heterogeneity distribution of the infinitesimal space (with hindering or obstacles)! However, substituting $d / d t$ by the fractional derivative $D_{t}^{\alpha}$ requires some considerations as we will see later in this paper.

Final Motivation. Most of analysis on fragmentation with first order derivative $d / d t$ has been performed under the assumption that the ensemble of particles is well mixed so that the particle distribution is uniform in space. However, recent approaches, using individual based models [18], yield in a natural way to systems in which splitting particles are distributed in space according to some prescribed probability density, leading, however, to models with explicit space dependence. We note that similar models were also considered earlier in [19] but with emphasis only on wellposedness. In this paper, the fractional version of the same type of model, namely, the fractional, nonlocal, and randomly position structured fragmentation model, is analyzed with full description given in the next section.

\section{Model Description and Settings}

2.1. Preliminaries: Classical Nonlocal and Randomly Position Structured Fragmentation Model. In the literature review on classical fragmentation models with "normal" time derivative of order one, they have been comprehensively analyzed in numerous works (see, e.g., [1-8]). Transport-type models with convection were investigated in [8] where the author showed that the convection part does not affect the breach of the conservation laws. In [2] the authors studied the nonlocal fragmentation and showed that the process is conservative if at infinity daughter particles tend to go back into the system with a high known probability. Honesty and nonconservative (dishonesty) regimes for fragmentation equations have been thoroughly investigated (see $[2,15])$ and, in particular, the breach of the mass conservation law (shattering) is believed to be beyond the model's resolution. The description of the dynamics of the classical nonlocal and randomly position structured fragmentation model is as follows: the state at a given time $t$ is the repartition at that time of all aggregates according to their size $m$ and their position $x$. In terms of $m$ and $x$, the state of the system is characterized at any moment $t$ by the particle-mass-position distribution $g=g(t, m, x)(g$ is also called the density or concentration of particles), where $g: \mathbb{R}_{+} \times \mathbb{R}_{+} \times \mathbb{R}^{3} \rightarrow \mathbb{R}_{+}$. Thus,

$$
\int_{n}^{p} \int_{\mathbb{R}^{3}} g(t, m, x) d x d m
$$

is the number of particles having mass between $n$ and $p$ and

$$
\int_{n}^{p} \int_{\mathbb{R}^{3}} g(t, m, x) m d x d m
$$

is the mass contained in particles in $\mathbb{R}^{3}$ having mass within this range.

Definition 1. The fragmentation rate $a=a(m, x)$ describes the ability of aggregates of size $m$ and position $x$ to break into smaller particles.

We assume that

$$
a \in L_{\infty, \text { loc }}\left(\mathbb{R}_{+} \times \mathbb{R}^{3}\right) .
$$

When an aggregate of mass $s$ located at $x$ breaks, the expected average number of daughter particles of size $m$ is a nonnegative measurable function $b(m, s, x)$ defined on $\mathbb{R} \times$ $\mathbb{R} \times \mathbb{R}^{3}$. Since a group of size $m \leq s$ cannot split to form 
a group of size $s$ then $\operatorname{Supp}(b) \subseteq\left\{(m, s) \in \mathbb{R}_{+} \times \mathbb{R}_{+}: m<\right.$ $s\} \times \mathbb{R}^{3}$ which yields

$$
b_{s, m}=0, \quad \forall m \leq s
$$

After the fragmentation of a mass $s$ particle, the sum of masses of all daughter particles should again be $s$; hence it follows that for any $s>0, x \in \mathbb{R}^{3}$

$$
\int_{0}^{s} n b(n, s, x) d n=s
$$

moreover, the quantity $\int_{0}^{s} b(n, s, x) d n$ gives us the expected number of daughter particles produced by fragmentation of a mass $s$ particle (with position $x$ ).

After a group's fragmentation, new originating daughter particles have different centers distributed according to a given probabilistic law $\tilde{b}(\cdot, m, s, y)$. This is the probability density that after a fragmentation of an $s$-aggregate (with the center at $y$ ) the new formed $m$-group will be located at the position $x$. Therefore

$$
\int_{\mathbb{R}^{3}} \widetilde{b}(x, m, s, y) d x=1 .
$$

This leads to the nonlocal and randomly position structured fragmentation model given by

$$
\begin{aligned}
\frac{\partial g}{\partial t}(t, m, x)= & -a(m, x) g(t, m, x) \\
& +\int_{m}^{\infty} \int_{\mathbb{R}^{3}} a(s, y) b(m, s, y) \\
& \times \tilde{b}(x, m, s, y) g(t, s, y) d y d s .
\end{aligned}
$$

The total mass of the ensemble at time $t$ is the quantity

$$
\int_{0}^{\infty} \int_{\mathbb{R}^{3}} g(t, m, x) m d x d m
$$

thus the natural space for analysis is

$$
X=L_{1}\left(\mathbb{R}_{+} \times \mathbb{R}^{3}, m d x d m\right) .
$$

In order to make use of the semigroup theory of linear operators we need to complement (9) with the initial massposition distribution

$$
g(0, m, x)=g_{0}(m, x), \quad \text { a.e }(m, x) \in \mathbb{R}_{+} \times \mathbb{R}^{3},
$$

where $g_{0} \in X$. In what follows we denote by $\mathscr{A}$ and $\mathscr{B}$ the expressions appearing on the right-hand side of (9); that is,

$$
\begin{gathered}
{[\mathscr{A} \psi](m, x)=-a(m, x) \psi(m, x)} \\
{[\mathscr{B} \psi](m, x)=\int_{m}^{\infty} \int_{\mathbb{R}^{3}} a(s, y) b(m, s, y)} \\
\quad \times \widetilde{b}(x, m, s, y) \psi(s, y) d y d s
\end{gathered}
$$

defined on all measurable and finite almost everywhere functions $\psi$ for which they make pointwise (almost everywhere) sense.

We introduce operators $A$ and $B$ in $X$ defined by

$$
[A u](m, x)=[\mathscr{A g}](m, x), \quad[B u](m, x)=[\mathscr{B g}](m, x)
$$

and set $D(A)=\{\psi \in X ; a \psi \in X\}$.

Lemma 2. $(A+B, D(A))$ is a well-defined operator.

Proof. In order to prove the first part of the theorem, we need to show that $\mathscr{B} D(A) \subset X$. Let $g \in D(A)_{+}$, changing the order of integration by the Fubini theorem; we obtain

$$
\begin{gathered}
\int_{0}^{\infty} \int_{\mathbb{R}^{3}} \mathscr{B} g(m, x) m d x d m \\
=\int_{\mathbb{R}^{3}} \int_{0}^{\infty}\left[\int_{m}^{\infty} a(s, y) b(m, s, y)\right. \\
\quad \times\left(\int_{\mathbb{R}^{3}} \tilde{b}(x, m, s, y) d x\right) \\
\times g(s, y) d s] m d m d y \\
=\int_{\mathbb{R}^{3}}\left(\int_{0}^{\infty} \int_{m}^{\infty} m a(s, y) b(m, s, y)\right. \\
=\int_{\mathbb{R}^{3}}\left(\int_{0}^{\infty} \int_{0}^{s} m a(s, y) b(m, s, y)\right. \\
\left.\left.=\int_{0}^{\infty} \int_{\mathbb{R}^{3}} a(s, y) g(s, y) s d y d s, y\right) d s d m\right) d y \\
\times g(s, y) d m d s) d y
\end{gathered}
$$

where we used (8) and (7), respectively. Because $g \in D(A)_{+}$ it follows that

$$
\int_{0}^{\infty} \int_{\mathbb{R}^{3}} \mathscr{B g}(m, x) m d x d m<+\infty .
$$

The result follows from the fact that any arbitrary element $g$ of $D(A)$ can be written in the form $g=g_{+}-g_{-}$, where $g_{+}, g_{-} \epsilon$ $D(A)_{+}$.

Let us define the terms stochastic and substochastic semigroups.

Definition 3. The strongly continuous semigroup of operators $(G(t))_{t \geq 0}$ on the Banach space $X$ is said to be

(i) substochastic if $S(t) \geq 0$ and $\|G(t)\| \leq 1$ for all $t \geq 0$,

(ii) stochastic if, in addition, it satisfies $\|G(t) \psi\|=\|\psi\|$ for all nonnegative $\psi \in X$. 
Usually, to find the semigroup for a given evolution equation, we use the Hille-Yosida theorem, stated as follows.

Theorem 4 (Hille-Yosida theorem). $\mathscr{P} \in \mathscr{G}(M, \omega)$ if and only if (a) $\mathscr{P}$ is closed and densely defined; (b) there exist $M>0$, $\omega \in \mathbb{R}$ such that $(\omega, \infty) \in \rho(\mathscr{P})$ and for all $n \geq 1, \lambda>\omega$

$$
\left\|(\lambda I-\mathscr{P})^{-n}\right\| \leq \frac{M}{(\lambda-\omega)^{n}},
$$

where $\rho(\mathscr{P})$ is the resolvent set of the operator $\mathscr{P}$ and is defined as follows:

$$
\begin{aligned}
\rho(\mathscr{P})=\{\lambda & \in \mathbb{R} ; \lambda I-\mathscr{P}: D(\mathscr{P}) \\
& \left.\longrightarrow X \text { is invertible and }(\lambda I-\mathscr{P})^{-1} \in \mathscr{L}(X)\right\} .
\end{aligned}
$$

Proof. The proof of this classic theorem can be found in any book of functional analysis. Note that $\mathscr{P} \in \mathscr{G}(M, \omega)$ means (see [8]) $\mathscr{P}$ generates a $C_{0}$-semigroup $\left(G_{\mathscr{P}}(t)\right)_{t>0}$ so that there exist $M>0$ and $\omega$ such that

$$
\left\|G_{\mathscr{P}}(t)\right\| \leq M e^{\omega t} .
$$

Then, we can state the following existence theorem for the classical nonlocal model which will help us in the analysis of the fractional model.

Theorem 5. Consider the model (9) expressed in terms of $A$ and $B$ defined in (14); then there is an extension $\widetilde{K}$ of $A+$ $B$ that generates a substochastic semigroup $\left(G_{\widetilde{K}}(t)\right)_{t \geq 0}$ on $X$. Moreover, for each $g_{0} \in D(\widetilde{K})$ there is a measurable representation $g(t, m, x)$ of $G_{\widetilde{K}}(t) g_{0}$ which is absolutely continuous with respect to $t \geq 0$ for almost any $(m, x)$ and such that $(9)$ is satisfied almost everywhere.

Proof. The proof was done in [3, Theorem 3] or [2, Theorem 3.3] for the discrete case of the same model.

\section{Solvability of the Fractional Nonlocal Model}

The fractional and nonlocal differential model of our interest is given by

$$
\begin{aligned}
D_{t}^{\alpha} g(t, m, x)= & -a(m, x) g(t, m, x) \\
& \quad+\int_{m}^{\infty} \int_{\mathbb{R}^{3}} a(s, y) b(m, s, y) \\
& \quad \times \widetilde{b}(x, m, s, y) g(t, s, y) d y d s
\end{aligned}
$$

with $(m, x) \in \mathbb{R}_{+} \times \mathbb{R}^{3}, 0<\alpha<1$, and $t>0$ or expressed in the abstract form as

$$
D_{t}^{\alpha} g(t)=[A+B] g(t), \quad 0<\alpha<1, t>0,
$$

with the assumption that it is subject to the initial condition

$$
g(0, m, x)=g_{0}(m, x), \quad(m, x) \in \mathbb{R}_{+} \times \mathbb{R}^{3}
$$

or simply

$$
g(0)=g_{0}
$$

Here, $A$ and $B$ are defined in (14) and $D_{t}^{\alpha}$ is defined by

$$
\begin{array}{r}
D_{\tau}^{\alpha} g(\tau, m, x)=-\lim _{t \rightarrow 0} \frac{\mathfrak{u}_{\alpha}(t) g(\tau, m, x)-g(\tau, m, x)}{t}, \\
x \in \mathbb{R}^{3}, \quad 0<\alpha<1, \quad t>0
\end{array}
$$

with $\mathfrak{t}_{\alpha}(t)$ the fractional time evolution (with index $\alpha, 0<$ $\alpha<1$ ) considered as universal attractor semigroups of coarse grained macroscopic time evolutions $[20,21]$. It is shown that $[20,22]$

$$
\begin{array}{r}
D_{\tau}^{\alpha} g(\tau, m, x)=-\frac{1}{\Gamma(-\alpha)} \int_{0}^{\infty} \frac{g(\tau-r, m, x)-g(\tau, m, x)}{r^{\alpha+1}} d r, \\
0<\alpha<1,
\end{array}
$$

which is the fractional derivative of the function $g$ in the sense of Michaud [22]. However, substituting $\partial_{t}$ by $D_{t}^{\alpha}$ in (9) is justified in the sense that the presence of $\partial_{t}$ in (9) reflects a basic symmetry of the time translation invariance and the basic principle of locality. Indeed, from the relation

$$
\frac{d}{d \tau} g(\tau)=\lim _{t \rightarrow 0} \frac{g(\tau)-g(\tau-t)}{t}=-\lim _{t \rightarrow 0} \frac{\mathfrak{u}(t) g(\tau)-g(\tau)}{t},
$$

we see that $-d / d t$ is identified as the infinitesimal generator of time translation $\mathfrak{u}(t) g(\tau)=g(\tau-t)$. Hence, this considers $\mathfrak{t}(t)$ as the expression of the general time evolution, which is the same consideration done in Definitions (24) and (25) of $D_{t}^{\alpha}$. Thus, the derivative of fractional order $D_{t}^{\alpha}, 0<\alpha<1$ was found to be, in general, infinitesimal generator of coarse grained macroscopic time evolution. It is well known [23] that all macroscopic time evolutions have fractional derivatives, with order less than unity, as their infinitesimal generators. Therefore, in Proposition 8, we provide a relation between the generator $D_{t}^{\alpha}, 0<\alpha<1$, of the macroscopic time evolution and $d / d t$.

Definition 6. Consider an operator $\mathscr{P}$ applied in the fractional model

$$
D_{t}^{\alpha} g(t, m, x)=\mathscr{P} g(t, m, x), \quad 0<\alpha<1, t>0, x \in \mathbb{R}^{3},
$$

subject to the initial condition

$$
g(x, 0)=f(x), \quad x \in \mathbb{R}^{3}
$$

and defined in the Banach space $X$. A family $\left(G_{\mathscr{P}}(t)\right)_{t>0}$ of bounded operators on $X$ is called a solution operator of the fractional Cauchy problem (27)-(28) if

(1) $G_{\mathscr{P}}(0)=I_{X}$;

(2) $G_{\mathscr{P}}(t)$ is strongly continuous for every $t \geq 0$; 
(3) $\mathscr{P}_{\mathscr{P}}(t) g=G_{\mathscr{P}}(t) \mathscr{P} g$ for all $g \in D(\mathscr{P})$;

(4) $G_{\mathscr{P}}(t) D(\mathscr{P}) \subset D(\mathscr{P})$;

(5) $G_{\mathscr{P}}(t) g$ is a (classical) solution of the model (27)-(28) for all $g \in D(P), t \geq 0$.

Recall that the operator $\widetilde{\mathscr{P}} \in \mathscr{G}(M, \omega)$ means $\widetilde{\mathscr{P}}$ generates a $C_{0}$-semigroup $\left(G_{\widetilde{P}}(t)\right)_{t>0}$ so that there exist $M>0$ and $\omega$ such that

$$
\left\|G_{\widetilde{P}}(t)\right\| \leq M e^{\omega t} .
$$

Whence, by analogy if the fractional Cauchy problem (27)(28) has a solution operator $\left(G_{\mathscr{P}}(t)\right)_{t>0}$ verifying (29), then we say that $\mathscr{P} \in \mathscr{G}^{\alpha}(M, \omega)$. The solution operator $\left(G_{\mathscr{P}}(t)\right)_{t>0}$ is contractive if

$$
\left\|G_{\mathscr{P}}(t)\right\|_{X} \leq 1,
$$

and we say $\mathscr{P} \in \mathscr{G}^{\alpha}(1,0)$.

Note that if we have a contraction solution operator, we can use Definition 6 to identify the fractional Cauchy problem of which it is a solution. Usually, however, we are interested in the reverse question, that is, in finding the solution operator that is contractive, for a given fractional model. The answer is given by the following theorem (seen as an analogue of Hille-Yosida Theorem 4).

Theorem 7. An operator $A_{\alpha} \in \mathscr{G}^{\alpha}(1,0)$ for $0<\alpha \leq 1$ if and only if

(a)

$$
(0, \infty) \subset \rho\left(A_{\alpha}\right)
$$

(b)

$$
\lambda^{\alpha-1} R\left(\lambda^{\alpha}, A_{\alpha}\right) f=\int_{0}^{\infty} e^{-\lambda r} G_{\alpha}(r) f d r,
$$

with $\lambda>0, f \in X$, where $\left(G_{\alpha}(t)\right)_{t>0}$ is a family of strongly continuous operators satisfying (30) and $\rho\left(A_{\alpha}\right)$ is the resolvent set of the operator $A_{\alpha}$ :

$$
\begin{aligned}
\rho\left(A_{\alpha}\right)=\{ & \left\{\lambda \in \mathbb{R} ; \lambda I-A_{\alpha}: D\left(A_{\alpha}\right) \longrightarrow Y_{1}\right. \\
& \text { is invertible and } \left.\left(\lambda I-A_{\alpha}\right)^{-1} \text { bounded and linear }\right\} .
\end{aligned}
$$

Proof. This theorem is a particular version of [24, Theorem 1.3] and the proof follows the same steps.

Consider the following problem:

$$
\begin{aligned}
D_{t}^{\alpha} g(t, m, x) & =-a(m, x) g(t, m, x) \\
( & =A g(t, m, x)), \quad 0<\alpha<1, \\
g(x, 0) & =f(x), \quad x \in \mathbb{R}^{3}, \quad t>0,
\end{aligned}
$$

with

$$
f \in D(A) .
$$

The full operator in the problem (21) is seen as a perturbation of operator $A$ in (34), which represents the loss part of the fractional fragmentation dynamics. Then, from the original semigroup $(G(t))_{t \geq 0}$ (the multiplication semigroup) generated by the differentiation operator $-d / d t$ and given after a simple integration by

$$
(G(t) g)(t, m, x)=e^{-a(m, x) t} g(t, m, x),
$$

one can exploit the theory of $C_{0}$-semigroup to state that the operator $A$ is the infinitesimal generator of a $C_{0}$-semigroup $\left(G_{A}(t)\right)_{t \geq 0}$. The existence of $(G(t))_{t \geq 0}$ comes from assumption that the fission rate satisfies (5). Therefore the infinitesimal generators of $\left(G_{A}(t)\right)_{t>0}$ may be interpreted [25] as the distributions, evaluated on the right time translation group, which leads to the following proposition.

Proposition 8. (a) $(G(t))_{t \geq 0}$ is an equibounded semigroup and the norms of all operators $G(t), t \in \mathbb{R}$ are bounded above by 1 .

(b) Let $g \in D(A)$. The infinitesimal generators $A$ of $C_{0}$-semigroups $\left(G_{A}(t)\right)_{t \geq 0}$ are related to $(S(t))_{t \geq 0}$ by the representations

$$
\begin{aligned}
&-\left(-\frac{d}{d t}\right)^{\alpha} g(x)=-D_{t}^{\alpha}(g(x)) \\
&=\lim _{\varepsilon \rightarrow 0+} \frac{1}{\Gamma(-\alpha)} \int_{\varepsilon}^{\infty} r^{-\alpha-1} \\
& \times\left[1-e^{-a(m, x) r}\right] g(x) d r,
\end{aligned}
$$

$\|G(t) g-g\| \leq M t^{\alpha}\|A u\| \quad$ for some $M>0$.

(c) For every $g \in D(A)$ one has $G_{A}(t) A u=A G_{A}(t) g$.

Proof. (a) By condition (5) we know (see $[1,2,8]$ ) that $A$ is the infinitesimal generator of a positive semigroup of contractions and the assertion follows.

To prove (b) we use Marchaud type representation [26] of fractional powers of infinitesimal generators. Let $\delta$ be the Dirac measure; then if we take $C_{0}^{1}(\mathbb{R})$ as the set of test functions on $\mathbb{R}$, we know that (see [27]) the fractional derivative distribution $\delta^{\alpha}, 0<\alpha<1$, of $\delta$ can be expressed by

$$
\left\langle\varphi, \delta^{\alpha}\right\rangle=\frac{1}{-\Gamma(-\alpha)} \int_{0}^{\infty} r^{-\alpha-1}[\varphi(x)-\varphi(x+r)] d r
$$

and approximated by the family $\left(\mathscr{M}_{\varepsilon}^{\alpha}\right)_{\varepsilon>0}$ of finite Borel measure on $\mathbb{R}_{+}$given as

$$
\begin{array}{r}
\left\langle\varphi, \mathscr{M}_{\varepsilon}^{\alpha}\right\rangle \\
=\frac{1}{-\Gamma(-\alpha)} \int_{\varepsilon}^{\infty} r^{-\alpha-1}[\varphi(x)-\varphi(x+r)] d r, \\
\forall \varphi \in C_{0}^{1}(\mathbb{R}) .
\end{array}
$$


The family $\left(\mathscr{M}_{\varepsilon}^{\alpha}\right)_{\varepsilon>0}$ generates the bounded linear operators $l_{\mathscr{M}_{\varepsilon}^{\alpha}}$ defined by

$$
\begin{array}{r}
l_{\mathscr{M}_{\varepsilon}^{\alpha}} g(x)=\frac{1}{-\Gamma(-\alpha)} \int_{\varepsilon}^{\infty} r^{-\alpha-1}\left[g(x)-G_{A}(r) g(x)\right] d r, \\
g \in X_{1} .
\end{array}
$$

Using Laplace transform $\mathscr{L}$, we can show (see [28]) that

$$
\begin{aligned}
& \mathscr{L}\left(l_{\mathscr{M}_{\varepsilon}^{\alpha}}\right)(x)=x^{\alpha} \cdot \mathscr{L}\left[\frac{1}{-\Gamma(-\alpha) \Gamma(1+\alpha)}\right. \\
&\left.\times\left(\left(\frac{\cdot}{\varepsilon}\right)_{+}^{\alpha}-\left(\frac{\dot{-}}{\varepsilon}+1\right)_{+}^{\alpha}\right)\right](x), \\
& \quad x>0,
\end{aligned}
$$

which yields, by uniqueness theorems for Laplace transformation and well known properties of convolution operator *,

$$
\mathscr{M}_{\varepsilon}^{\alpha}=\delta^{\alpha} *\left[\frac{1}{-\Gamma(-\alpha) \Gamma(1+\alpha)}\left(\left(\frac{\cdot}{\varepsilon}\right)_{+}^{\alpha}-\left(\frac{\dot{-}}{\varepsilon}+1\right)_{+}^{\alpha}\right)\right]
$$

and then

$$
\begin{gathered}
A \int_{0}^{\infty} \frac{1}{-\Gamma(-\alpha) \Gamma(1+\alpha)}\left(\left(\frac{r}{\varepsilon}\right)_{+}^{\alpha}-\left(\frac{r}{\varepsilon}+1\right)_{+}^{\alpha}\right) G_{A}(r) g(x) d r \\
=\frac{1}{-\Gamma(-\alpha)} \int_{\varepsilon}^{\alpha} r^{-\alpha-1}\left[g(x)-G_{A}(r) g(x)\right] d r .
\end{gathered}
$$

Now taking $\varepsilon \rightarrow 0$, the assertion (b) follows by using the fact that $A_{\alpha}$ is a closed operator [29] and

$$
\begin{aligned}
\lim _{\varepsilon \rightarrow 0} \int_{0}^{\infty} \frac{1}{-\Gamma(-\alpha) \Gamma(1+\alpha)}\left(\left(\frac{r}{\varepsilon}\right)_{+}^{\alpha}-\left(\frac{r}{\varepsilon}+1\right)_{+}^{\alpha}\right) G_{A}(r) \\
\quad \times g(x) d r=g(x) .
\end{aligned}
$$

To prove (39) and (c), we make use of [30, Theorem 6.13 p.74]. We just need to show that $0 \in \rho(A)$. In fact since $a \in L_{\infty, \text { loc }}(0, \infty)$ then $0 \notin \overline{a((0, \infty))}$; hence, $a$ has abounded inverse $1 / a$ and so does $A$. Thus $0 \in \rho(A)$, which completes the proof.

Let us now consider the Mittag-Leffler relaxation function $E_{\alpha}(-x)$, where $x$ is a positive real number (time variable for example) and used to describe complex relaxation processes. We have that $[31,32]$

$$
E_{\alpha}[z]=\sum_{n=0}^{\alpha} \frac{z^{n}}{\Gamma(n \alpha+1)}
$$

where $\Gamma$ is the Gamma function. Using the Laplace transform $\mathscr{L}$ and its inverse, it can be proved (see [33-35]) that

$$
\begin{aligned}
E_{\alpha}(-x) & =\frac{2 x}{\pi} \int_{0}^{\infty} \frac{E_{2 \alpha}\left(-s^{2}\right)}{x^{2}+s^{2}} d s \\
& =\frac{\sin (\alpha \pi)}{\pi} \int_{0}^{\infty} \frac{s^{\alpha-1} e^{-x^{1 / \alpha} s} d s}{s^{2 \alpha}+2 s^{\alpha} \cos (\alpha \pi)+1} \\
& =\int_{0}^{\infty} \sum_{n=0}^{\infty} \frac{(-s)^{n} e^{-x s} d s}{n ! \Gamma(1-\alpha-\alpha n)} \\
& =\frac{1}{2 \pi i} \int_{\Upsilon} e^{\left(s-x s^{\alpha}\right)} s^{\alpha-1} e^{-x s} d s, \quad 0<\alpha<1,
\end{aligned}
$$

where $\Upsilon$ is a contour domain which encircles the origin counterclockwise, going from $-\infty$ to $-\infty$.

Theorem 9. There is a positive and contractive solution operator $\left(G_{K}(t)\right)_{t>0}$ to the fractional model (21), where $K \in$ $\mathscr{G}^{\alpha}(1,0)$ is an extension of the operator sum $A+B$ defined in (14).

Proof. Consider the classical model (9) given as

$$
\begin{gathered}
\frac{d}{d t} g(t, m, x)=[A+B] g(t, m, x), \\
g(0)=g_{0},
\end{gathered}
$$

where $A$ and $B$ are defined in (14). This model is the same as

$$
\begin{gathered}
D_{t}^{1} g(t, m, x)=[A+B] g(t, m, x), \\
g(0)=g_{0} .
\end{gathered}
$$

Then, by Theorem 5 , there is an extension $\widetilde{K}$ of the operator $A+B$ which generates a positive semigroup of contractions $\left(G_{\widetilde{K}}(t)\right)_{t \geq 0}$ and, then, satisfies

$$
\left\|G_{\widetilde{K}}(t)\right\|_{X} \leq 1 .
$$

Now, using Definition 6, $\widetilde{K} \in \mathscr{G}(1,0)$. Exploiting the relaxation relation $(48)$, we set

$$
G_{K}(t) f=\int_{0}^{\infty} \Psi_{\alpha}(t, s) G_{\widetilde{K}}(s) f d s,
$$

where

$$
\Psi_{\alpha}(t, s)=\frac{1}{t^{\alpha}} \sum_{n=0}^{\infty} \frac{(-s)^{n} t^{-\alpha n}}{n ! \Gamma(1-\alpha-\alpha n)} .
$$

We aim to show that $G_{K}(t)$ is the solution operator for the fractional model (21) and is positive and contractive. The second last relation of (48) and monotonicity of $E_{\alpha}(-x)$ imply that $\left(G_{K}(t)\right)_{t>0}$ is positive. By Hille-Yosida Theorem 4, we have $(0, \infty) \subset \rho(K)$ and $\left\|G_{K}(s)\right\|_{X} \leq 1, s \geq 0$ since $G_{K}(t)$ is substochastic. Then using (48) and the fact (see $[34,35]$ ) that

$$
\int_{0}^{\infty}\left(\sum_{n=0}^{\infty} \frac{(-s)^{n}}{n ! \Gamma(1-\alpha-\alpha n)}\right) d s=1
$$


yield $\|G(s)\|_{X} \leq \int_{0}^{\infty} \Psi_{\alpha}(t, s)\left\|G_{K}(s)\right\|_{X} d s \leq 1, t \geq 0$; hence $(G(t))_{t>0}$ is contractive. To conclude the theorem, we make use of the subordination principle $[24,36]$ to state that $\widetilde{K} \in$ $\mathscr{G}^{\alpha}(1,0)$ since $\widetilde{K} \in \mathscr{G}(1,0)$. Thus, by $(52)$ and making use of Theorem 7, we have (see also [36, Theorem 3.1]) that $G_{K}(t)$ verifies $\left\|G_{K}(t)\right\|_{X} \leq 1$ and is the solution operator for the fractional model (21), that is, positive and contractive.

\section{Conclusion and Possible Future Analysis}

In this paper, we proved that there is a strongly continuous solution operator, positive and contractive, to nonlocal fragmentation models with Michaud time derivative of fractional order. To obtain this existence result, we made use of the subordination principle for differential equations of fractional order together with the theory of substochastic semigroup, the analogue of Hille-Yosida theorem for fractional model, and properties of Mittag-Leffler relaxation function. This existence result extends the preceding ones with the inclusion of the position dependence on the fragmentation rate and the derivative of order less than unity. Thus, more analysis can be done on the existence of the solution operator to the model and the study of its characteristics (strong continuity, positivity, and contractivity) may lead to a better understanding of strange observed phenomena like the sudden appearance of an infinite number of particles in some systems with initial finite particles number and the shattering phenomenon. The results in the present paper may also lead to the full characterization of the operator under which the fractional model admits a solution operator.

\section{Conflict of Interests}

The authors declare that there is no conflict of interests regarding the publication of this paper.

\section{References}

[1] E. F. Doungmo Goufo and S. C. Oukouomi Noutchie, "Global analysis of a discrete non-local and non-autonomous fragmentation dynamics occurring in a moving process," Abstract and Applied Analysis, vol. 2013, Article ID 484391, 9 pages, 2013.

[2] E. F. Doungmo Goufo and S. C. Oukouomi Noutchie, "Honesty in discrete, nonlocal and randomly position structured fragmentation model with unbounded rates," Comptes Rendus Mathematique: Comptes Rendus de l'Académie des Sciences I, vol. 351, no. 19-20, pp. 753-759, 2013.

[3] S. C. Oukouomi Noutchie and E. F. Doungmo Goufo, "On the honesty in nonlocal and discrete fragmentation dynamics in size and random position," ISRN Mathematical Analysis, vol. 2013, Article ID 908753, 7 pages, 2013.

[4] R. M. Ziff and E. D. McGrady, "The kinetics of cluster fragmentation and depolymerisation," Journal of Physics A, vol. 18, no. 15, pp. 3027-3037, 1985.

[5] C. R. Garibotti and G. Spiga, "Boltzmann equation for inelastic scattering," Journal of Physics A, vol. 27, no. 8, pp. 2709-2717, 1994.

[6] A. Majorana and C. Milazzo, "Space homogeneous solutions of the linear semiconductor Boltzmann equation," Journal of
Mathematical Analysis and Applications, vol. 259, no. 2, pp. 609629, 2001.

[7] W. Wagner, "Explosion phenomena in stochastic coagulationfragmentation models," Annals of Applied Probability, vol. 15, no. 3, pp. 2081-2112, 2005.

[8] S. C. Oukouomi Noutchie and E. F. Doungmo Goufo, "Global solvability of a continuous model for nonlocal fragmentation dynamics in a moving medium," Mathematical Problems in Engineering, vol. 2013, Article ID 320750, 8 pages, 2013.

[9] M. Caputo, "Linear models of dissipation whose Q is almost frequency independent: part II," Geophysical Journal International, vol. 13 , no. 5, pp. 529-539, 1967.

[10] K. B. Oldham and J. Spanier, The Fractional Calculus, Academic Press, New York, NY, USA, 1999.

[11] I. Podlubny, Fractional Differential Equations, vol. 198, Academic Press, San Diego, Calif, USA, 1999.

[12] K. S. Miller and B. Ross, An Introduction to the Fractional Calculus and Fractional Differential Equations, John Wiley \& Sons, New York, NY, USA, 2003.

[13] A. Atangana and J. F. Botha, "A generalized groundwater flow equation using the concept of variable order derivative," Boundary Value Problems, vol. 2013, article 53, 2013.

[14] A. Atangana and P. D. Vermeulen, "Analytical solutions of a space-time fractional derivative of groundwater flow equation," Abstract and Applied Analysis, vol. 2014, Article ID 381753, 11 pages, 2014.

[15] R. M. Ziff and E. D. McGrady, "'Shattering' transition in fragmentation," Physical Review Letters, vol. 58, no. 9, pp. 892-895, 1987.

[16] W. J. Anderson, Continuous-Time Markov Chains. An Applications-Oriented Approach, Springer, New York, NY, USA, 1991.

[17] J. R. Norris, Markov Chains, Cambridge University Press, Cambridge, UK, 1998.

[18] R. Rudnicki and R. Wieczorek, "Phytoplankton dynamics: from the behaviour of cells to a transport equation," Mathematical Modelling of Natural Phenomena, vol. 1, no. 1, pp. 83-100, 2006.

[19] M. Lachowicz and D. Wrzosek, "A nonlocal coagulationfragmentation model," Applicationes Mathematicae, vol. 27, no. 1, pp. 45-66, 2000.

[20] A. V. Balakrishnan, "Fractional powers of closed operators and the semigroups generated by them," Pacific Journal of Mathematics, vol. 10, pp. 419-437, 1960.

[21] K. Yosida, Functional Analysis, Springer, New York, NY, USA, 6th edition, 1980.

[22] S. G. Samko, A. A. Kilbas, and O. I. Marichev, Franctional Integrals and Derivatives. Theory and Application, Gordon and Breach Science, Amsterdam, The Netherlands, 1993.

[23] R. Hilfer, On New Class of Phase Transitions, Random Magnetism and High Temprature Superconductivity, World Scientific, Singapore, 1994.

[24] J. Prüss, Evolutionary Integral Equations and Applications, Birkhäuser, Basel, Switzerland, 1993.

[25] I. M. Gel'fand and G. Shilov, Generalized Functions, vol. 1, Academic Press, New York, NY, USA, 1964.

[26] J. L. Lions and J. Peetre, "Sur une classe d'espaces d'interpolation," Publications Mathématiques de l'Institut des Hautes Études Scientifiques, vol. 19, no. 1, pp. 5-68, 1964.

[27] B. Rubin, Fractional Integrals and Potentials, Addison-Wesley, Longman, Harlow, UK, 1996. 
[28] U. Westphal, "Ein Kalkül für gebrochene Potenzen infinitesimaler Erzeuger von Halbgruppen und Gruppen von Operatoren, Teil I: Halbgruppen-erzeuger," Compositio Mathematica, vol. 22, pp. 67-103, 1970.

[29] K.-J. Engel and R. Nagel, One-Parameter Semigroups for Linear Evolution Equations, Graduate Texts in Mathematics (Book 194), Springer, New York, NY, USA, 2000.

[30] A. Pazy, Semigroups of Linear Operators and Applications to Partial Differential Equations, vol. 44 of Applied Mathematical Sciences, Springer, New York, NY, USA, 1983.

[31] G. M. Mittag-Leffler, "Sur la nouvelle fonction E $\alpha(x)$," Comptes Rendus Mathematique: Comptes Rendus de l'Académie des Sciences II, vol. 137, pp. 554-558, 1903.

[32] A. Erdélyi, W. Magnus, F. Oberhettinger, and F. G. Tricomi, Higher Transcendental Functions, vol. 3, McGraw-Hill, New York, NY, USA, 1955.

[33] M. N. Berberan-Santos, "Properties of the Mittag-Leffler relaxation function," Journal of Mathematical Chemistry, vol. 38, no. 4, pp. 629-635, 2005.

[34] R. Gorenflo, Y. Luchko, and F. Mainardi, "Analytical properties and applications of the Wright function," Fractional Calculus \& Applied Analysis, vol. 2, no. 4, pp. 383-414, 1999.

[35] E. M. Wright, "The generalized Bessel function of order greater than one," The Quarterly Journal of Mathematics, vol. 11, pp. 3648, 1940.

[36] E. G. Bazhlekova, "Subordination principle for fractional evolution equations," Fractional Calculus \& Applied Analysis, vol. 3, no. 3, pp. 213-230, 2000. 


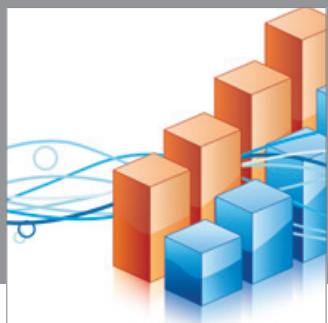

Advances in

Operations Research

mansans

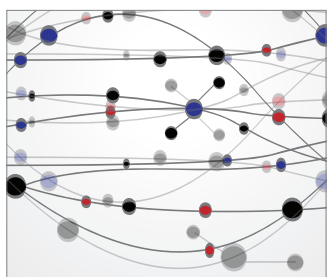

The Scientific World Journal
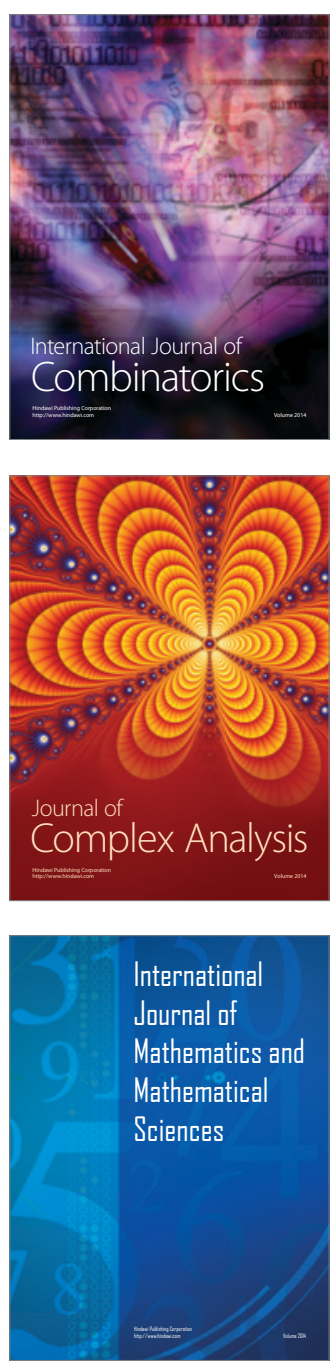
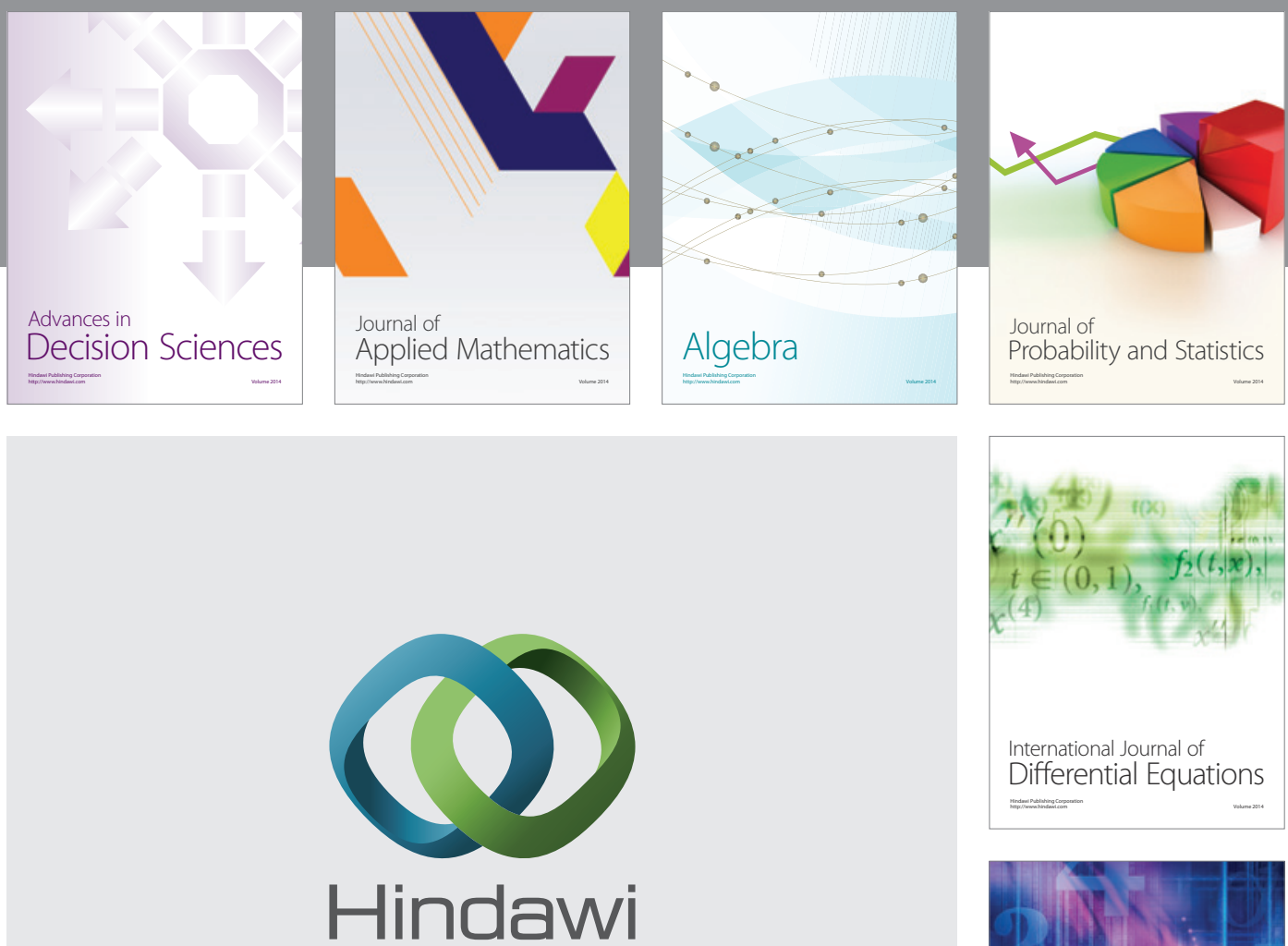

Submit your manuscripts at http://www.hindawi.com
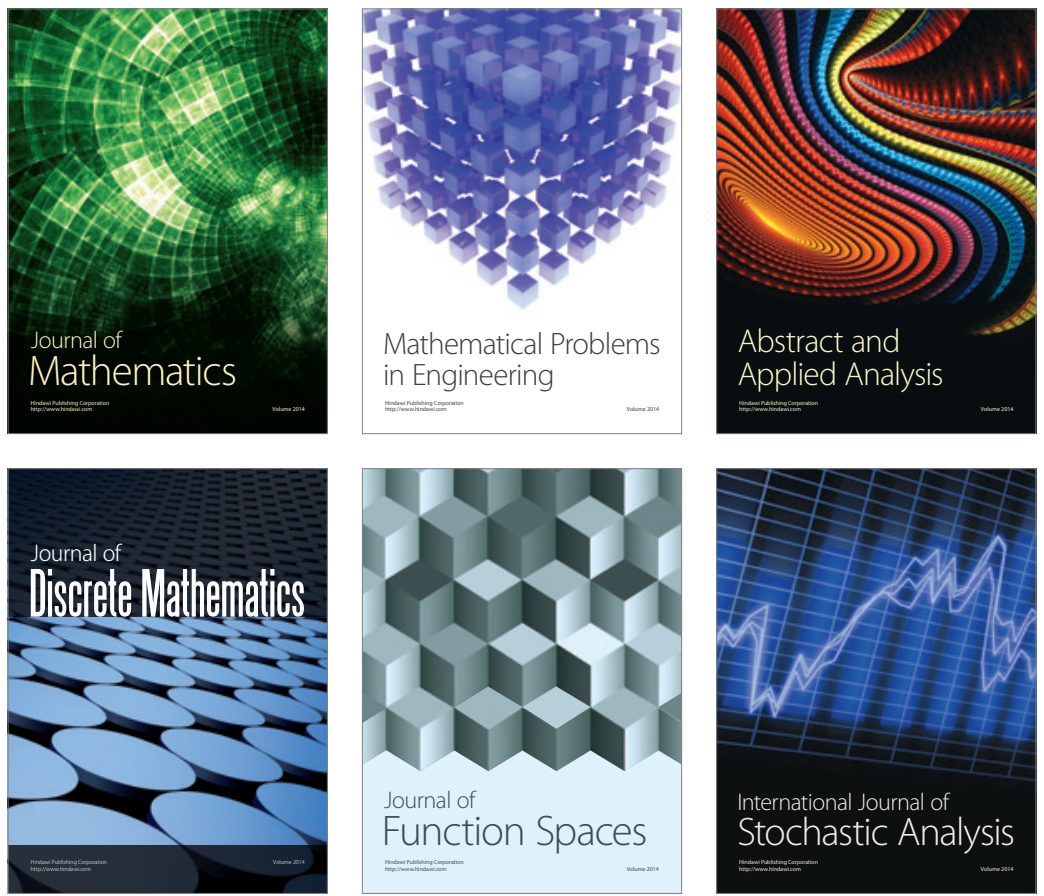

Journal of

Function Spaces

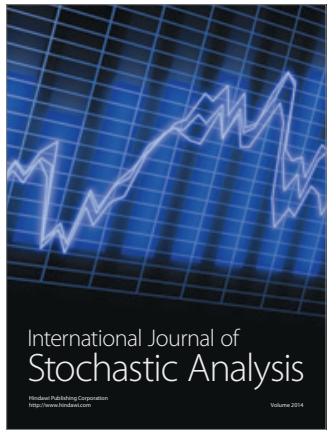

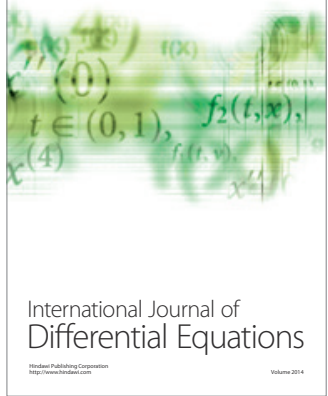
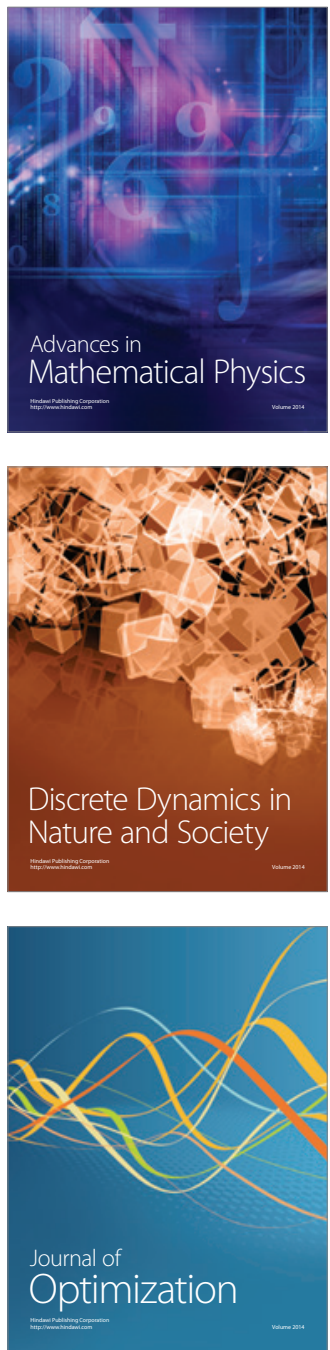